Research Article

\title{
Extended Holographic Ricci Dark Energy in Chameleon Brans-Dicke Cosmology
}

\author{
Surajit Chattopadhyay \\ Pailan College of Management and Technology, Bengal Pailan Park, Kolkata 700 104, India \\ Correspondence should be addressed to Surajit Chattopadhyay; surajitchatto@gmail.com
}

Received 7 August 2013; Accepted 17 September 2013

Academic Editors: W. Li and M. Tórtola

Copyright ( 2013 Surajit Chattopadhyay. This is an open access article distributed under the Creative Commons Attribution License, which permits unrestricted use, distribution, and reproduction in any medium, provided the original work is properly cited.

In the present work, we have studied some features of the generalized Brans-Dicke (BD) model in which the scalar field is allowed to couple nonminimally with the matter sector. Extended holographic Ricci dark energy (EHRDE) has been considered in the above framework of BD cosmology. Some restrictions have been derived for the BD parameter $\omega$, and a stronger matter-chameleon coupling has been observed with the expansion of the universe. In this framework, the equation of the state parameter of EHRDE has behaved like quintom. Also, we have reconstructed the potential and coupling function for BD model for the EHRDE. It has been observed that the potential function is increasing as the matter-chameleon coupling is getting stronger.

\section{Introduction}

Accumulating the observational data of Supernovae Type Ia (SN Ia) by the year 1998, Riess et al. [1] in the High-redshift Supernova Search Team and Perlmutter et al. [2] in the Supernova Cosmology Project Team have independently reported that the present universe is accelerating. The source for this late-time acceleration was dubbed "dark energy" (DE), which is distinguished from ordinary matter species such as baryons and radiation, in the sense that it has a negative pressure. Reviews on DE are available in [3-9]. The SN Ia observations have shown that about $70 \%$ of the present energy of the universe consists of DE [7]. The simplest candidate for $\mathrm{DE}$ is the so-called cosmological constant $\Lambda$, whose energy density remains constant [10]. If the origin of $\mathrm{DE}$ is not the cosmological constant, then one may seek for some alternative models for DE to explain the cosmic acceleration today. There are two approaches to construct models of DE other than cosmological constant [7]: (i) to modify the right-hand side of the Einstein equations by considering specific forms of energymomentum tensor $T_{\mu \nu}$ with negative pressure; (ii) to modify the left-hand side of the Einstein equations. The representative models that belong to the first category are the so-called quintessence [11], k-essence [12], and perfect fluid models [13]. The representative models that belong to the second class are "modified gravity" [14-22] models that include $f(R)$ gravity [19], scalar-tensor theories [20], and braneworld models [21]. Among all scalar-tensor theories of gravity, the simplest one is the so-called Brans-Dicke theory (BD) introduced by Brans and Dicke [23] that modifies general relativity in accordance with Mach's principle. Cosmological models of classical $\mathrm{BD}$ theory were first studied in $[24,25]$. In the subsequent decades, various aspects of $\mathrm{BD}$ cosmology have been widely investigated [26-34].

The present paper is aimed at working in chameleon Brans-Dicke cosmology. Thus, we discuss a bit of chameleon cosmology in this place. Khoury and Weltman $[35,36]$ employed self-interactions of the scalar field to avoid the bounds on such a field and dubbed such scalars to be "chameleon fields" due to the way in which the field's mass depends on the density of matter in the local environment. In regions of high density, the chameleon "blends" with its environment and becomes essentially invisible to search for the violation of equivalence principle and the fifth force [35]. Reference [37] has shown that chameleon scalar field can provide explicit realizations of a quintessence model where the quintessence 
scalar field couples directly to baryons and dark matter with gravitational strength. Reference [38] has illustrated that interacting chameleon field plays an important role in latetime universe acceleration and phantom crossing. Reference [39] proposed an interacting holographic dark energy model in chameleon-tachyon cosmology by interaction between the components of the dark sectors. Based on two independent functions of the scalar field, [40] constructed an exact solution describing the evolution of the type Bang-to-Rip with the phantom divide line crossing in the chameleon cosmology.

Now, we come to the chameleon Brans-Dicke cosmology. In the models, where a nonminimal coupling between the scalar field and matter system is considered by introducing an arbitrary functions of the scalar field, the scalar field is regarded as as a chameleon field [33]. Reference [41] was the first to introduce this kind of chameleon-matter coupling in the $\mathrm{BD}$ model to achieve accelerated the expansion of the universe. In a recent paper, [33] considered a generalized BD model with power-law form of the scale factor and the coupling functions as the inputs and allowed nonminimal coupling with the matter sector to show that accelerated expansion of the universe can be realized for a contrained range of exponents of the potential function. Treating the BD scalar field as a chameleon scalar field and taking a nonminimal coupling of the scalar field with matter, [42] studied cosmological implication of holographic dark energy in the $\mathrm{BD}$ gravity. Reference [43] investigated the cosmological applications of interacting holographic dark energy in $\mathrm{BD}$ theory with chameleon scalar field which is nonminimally coupled to the matter field and observed that phantom crossing can be constructed if the model parameters are chosen suitably. For different epochs of the cosmic evolution, [44] investigated the $\mathrm{BD}$ chameleon theory of gravity and obtained exact solutions of the scale factor and scalar field and potential. Reference [45] discussed late-time dynamics of a chameleonic generalized BD cosmology with the power law chameleonic function $f(\phi) \propto \phi^{n}$, where $n$ is a real parameter motivated from string theories.

Making an approach to dark energy in the frame of quantum gravity is the well-known holographic dark energy (HDE) inspired by the holographic principle [46]. The HDE, whose density is $\rho_{\Lambda}=3 c^{2} M_{p}^{2} L^{-2}$ was proposed by Li [47]. Subsequent studies on HDE from various points of view include [48-51]. In the present work, we are considering a special form of HDE [52] dubbed as "extended holographic Ricci dark energy" (EHRDE) [53], whose density has the form

$$
\rho_{\Lambda}=3 M_{p}^{2}\left(\alpha H^{2}+\beta \dot{H}\right)
$$

where $M_{p}^{2}$ is the reduced Planck mass and $\alpha$ and $\beta$ are constants to be determined. Like [42], we will assume that $\rho_{\Lambda}$ and pressureless dark matter are conserved separately, and we will assume a nonminimal coupling between the scalar field and the matter field.

\section{EHRDE in Chameleon BD Cosmology}

We begin with the chameleon BD theory in which the scalar field is coupled nonminimally to the matter field via the action [33]

$$
S=\frac{1}{2} \int d^{4} x \sqrt{-g}\left(\phi R-\frac{\omega}{\phi} g^{\mu \nu} \nabla_{\mu} \phi \nabla_{\nu} \phi-2 V+2 f(\phi) L_{m}\right) .
$$

In (2), $R$ and $\phi$ denote the Ricci scalar field and BD scalar field, respectively. The $f(\phi)$ and $V(\phi)$ are analytic functions of the scalar field. The matter Lagrangian density, denoted by $L_{m}$, is coupled with $\phi$ via the function $f(\phi)$. This function allows a nonminimal coupling between the matter system and the scalar field. If $f(\phi)=1$, we get back the BD action with potential function $\phi$ [33]. Varying the action with respect to the metric $g_{\mu \nu}$ and $\phi$, one gets the field equations [33]

$$
\begin{gathered}
\phi G_{\mu \nu}=T_{\mu \nu}^{\phi}+f(\phi) T_{\mu \nu}^{m}, \\
(2 \omega+3) \square \phi+2\left(2 V-V^{\prime} \phi\right)=T^{m} f-2 f^{\prime} \phi_{m},
\end{gathered}
$$

where $\square=\nabla^{\mu} \nabla_{\mu} \cdot \nabla_{\mu}$ represents the covariant derivative, $T^{m}=$ $g^{\mu \nu} T_{\mu \nu}^{m}$, and the prime denotes differentiation with respect to $\phi$. In (3),

$$
\begin{aligned}
T_{\mu \nu}^{\phi}= & \frac{\omega}{\phi}\left(\nabla_{\mu} \phi \nabla_{\nu} \phi-\frac{1}{2} g_{\mu \nu} \nabla_{\alpha} \phi \nabla^{\alpha} \phi\right) \\
& +\left(\nabla_{\mu} \nabla_{\nu} \phi-g_{\mu \nu} \square \phi\right)-V(\phi) g_{\mu \nu}, \\
& T_{\mu \nu}^{m}=\frac{-2}{\sqrt{-g}} \frac{\delta\left(\sqrt{-g} L_{m}\right)}{\delta g^{\mu \nu}} .
\end{aligned}
$$

Because of the explicit coupling between matter system and $\phi$, the stress tensor $T_{\mu \nu}^{m}$ is not divergence free.

Now, we will apply the above framework to a homogeneous and isotropic universe described by the FriedmanRobertson-Walker metric

$$
d s^{2}=-d t^{2}+a^{2}(t)\left(\frac{d r^{2}}{1-k r^{2}}+r^{2} d \Omega^{2}\right) .
$$

The universe is open, closed, or flat according to $k=-1,+1$ or 0 . In a spatially flat universe, (3) and (6) yield

$$
\begin{aligned}
3 H^{2} & =\frac{f}{\phi} \rho+\frac{\omega}{2} \frac{\dot{\phi}^{2}}{\phi^{2}}-3 H \frac{\dot{\phi}}{\phi}+\frac{V}{\phi} \\
3\left(\dot{H}+H^{2}\right)= & -\frac{3 \rho}{\phi(2 \omega+3)}\left[\gamma \phi f^{\prime}+\left(\omega\left(\gamma+\frac{1}{3}\right)+1\right) f\right] \\
& -\omega \frac{\dot{\phi}^{2}}{\phi^{2}}+3 H \frac{\dot{\phi}}{\phi}+\frac{1}{2 \omega+3} \\
& \times\left[3 V^{\prime}+(2 \omega-3) \frac{V}{\phi}\right] \\
(2 \omega & +3)(\ddot{\phi}+3 H \dot{\phi})-2\left(2 V-\phi V^{\prime}\right) \\
& =\rho\left((1-3 \gamma) f+2 \gamma \phi f^{\prime}\right)
\end{aligned}
$$


In (8) and (10), $\rho=\rho_{m}+\rho_{\Lambda}$ and $\gamma=p_{\Lambda} /\left(\rho_{m}+\rho_{\Lambda}\right)$ (since dark matter is pressureless, $\left.p_{m}=0\right)$. This approach is similar to $[42,43]$.

In the present work, we will investigate two cases. In one case, we will assume special forms for $V, f, a$, and $\phi$ and derive conditions that strengthen matter-chameleon coupling with the expansion of the universe. In another case, we will not assume any form for $V$ and $f$. Rather we will reconstruct them for the EHRDE.

\section{Discussion}

3.1. Case I. In this section, we will consider a set of ansatz for the potential function, analytic function, scalar field, and scale factor. Based on them, we will determine constraint on the BD parameter $\omega$. Subsequently, based on the constraints on $\omega$ and other parameters, we will investigate the behavior of the EHRDE in BD chameleon cosmology. Following [33], we choose

$$
\begin{array}{cc}
V(\phi)=V_{0} \phi^{l_{1}} ; & f(\phi)=f_{0} \phi^{l_{2}} ; \\
a(t)=a_{0} t^{n} ; & \phi(t)=\phi_{0} t^{m} .
\end{array}
$$

Since $M_{p}^{2}=1 / 8 \pi G$ and in BD theory $\phi \propto G^{-1}$ [43], we can take EHRDE in the chameleon BD as

$$
\rho_{\Lambda}=3 \phi\left(\alpha H^{2}+\beta \dot{H}\right) \text {. }
$$

And using conservation equation $\dot{\rho}_{\Lambda}+3 H\left(\rho_{\Lambda}+p_{\Lambda}\right)=0$, we get

$$
p_{\Lambda}=\phi\left(\left(1-3 H^{2}-2 \dot{H}\right) \alpha+\frac{\beta}{H^{2}}(\dot{H}-H(\ddot{H}+3 H \dot{H}))\right) .
$$

Using (11)-(14) in (8), we have

$$
\varrho_{1} t^{m l_{2}-m+2-3 n}+\varrho_{2} t^{2-m+m l_{1}}+\varrho_{3} t^{m l_{2}}=\varrho_{4},
$$

where $\varrho_{1}, \varrho_{2}, \varrho_{3}$, and $\varrho_{4}$ are terms involving constant terms of ansatz (11). In (15), we observe that the right-hand side is constant with $t$ involved in the left-hand side. This is possible if the powers of $t$ are $0 . \operatorname{In}(15), m l_{2} \neq 0$. However, its coefficient $3 n f_{0} \phi_{0}^{l_{2}}(n \alpha-\beta)\left(3+\omega+3 \gamma\left(l_{2}+\omega\right)\right)$ can be set equal to 0 . Since $n \alpha-\beta=0$ will lead to $p_{\Lambda}=0$, we can set $3+\omega+3 \gamma\left(l_{2}+\omega\right)=0$. Finally, we get the restrictions

$$
\begin{gathered}
\omega=-\frac{3\left(1+\gamma l_{2}\right)}{1+3 \gamma}, \\
-2=m\left(l_{2}-1\right)-3 n, \\
-2=m\left(l_{1}-1\right) .
\end{gathered}
$$

The above restrictions are also found valid if we use the ansatz (11) and (13)-(14) in (9). In (16), we have for the accelerated expansion of the universe $3 \gamma+1>0$. Hence, (16) finally leads to a constraint on the $\mathrm{BD}$ parameter as

$$
\omega>\frac{l_{2}-3}{1+3 \gamma} \text {. }
$$

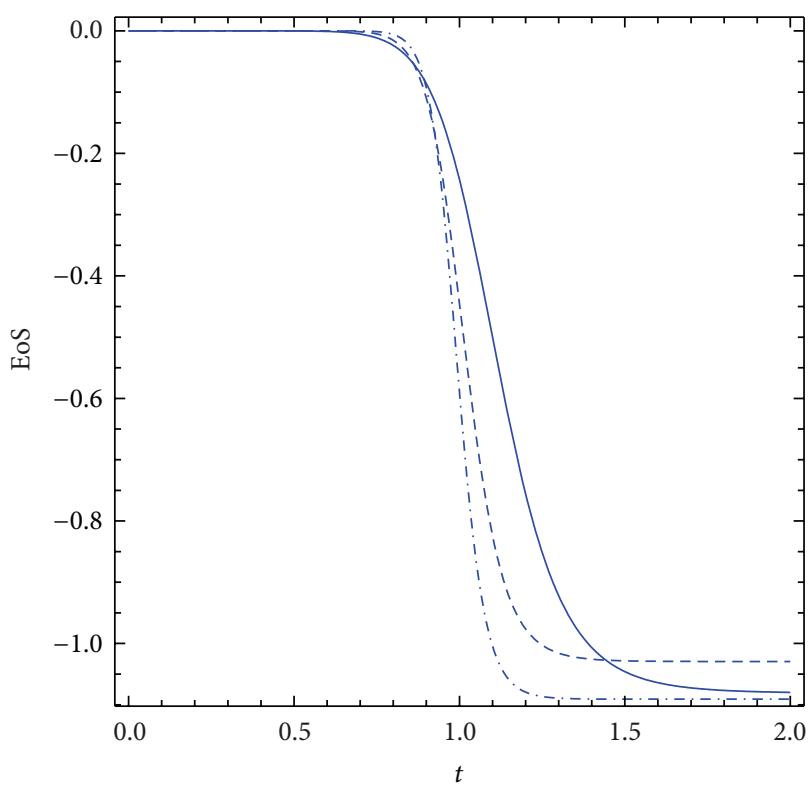

FIGURE 1: Evolution of EoS parameter $\gamma$ against $t$. The solid, dotdashed and dashed lines have the parameter combinations $\alpha=$ $2.3,1.3,1.5, \beta=4.5,2.5,1.5, l_{1}=0.3,0.5,0.2$, and $l_{2}=4.5,6,7$.

In 1973, $\omega>5$ was consistent with the known data. By 1981, $\omega>30$ was consistent with the known data [54]. In 2003, evidence derived from the Cassini-Huygens experiment shows that the value of $\omega$ must exceed 40,000 [55]. Hence, we can rewrite

$$
\omega>\frac{l_{2}-3}{1+3 \gamma} \gg 1 \text {. }
$$

Hence, $l_{2}>4$. From (17), we have

$$
n=\frac{2}{3}\left(\frac{l_{1}-l_{2}}{l_{1}-1}\right) ; \quad m=\frac{2}{1-l_{1}} .
$$

If $l_{1}>1$, then $m<0$, which implies that the scalar field is a decreasing function of $t$. Since $n$ has to be positive, $l_{1}>l_{2}$ from (20). Again, if $l_{1}<1$, then $m>0$. Since $l_{2}>0, m l_{2}>0$. Thus, for $l_{1}<1$, coupling function $f \propto t^{m l_{2}}$ increases with time, that is, matter-chameleon coupling gets stronger as the universe expands.

Choosing appropriate parameters (keeping the restrictions in mind), we have plotted the EoS parameter $\gamma=$ $p / \rho$ for different combinations of parameters in Figure 1. The EoS parameter clearly shows a transition from $\gamma>-1$ (quintessence) to $\gamma<-1$ (phantom). Hence, the EoS parameter is found to exhibit "quintom"-like behavior. Next, we plot in Figure 2 the deceleration parameter $q$ [53]

$$
q=\frac{1}{2}+\frac{3 p}{2 \rho}
$$

We observe that the deceleration parameter has a clear transition from positive to negative side. This indicates the transition from decelerated to accelerated phase of the universe. 


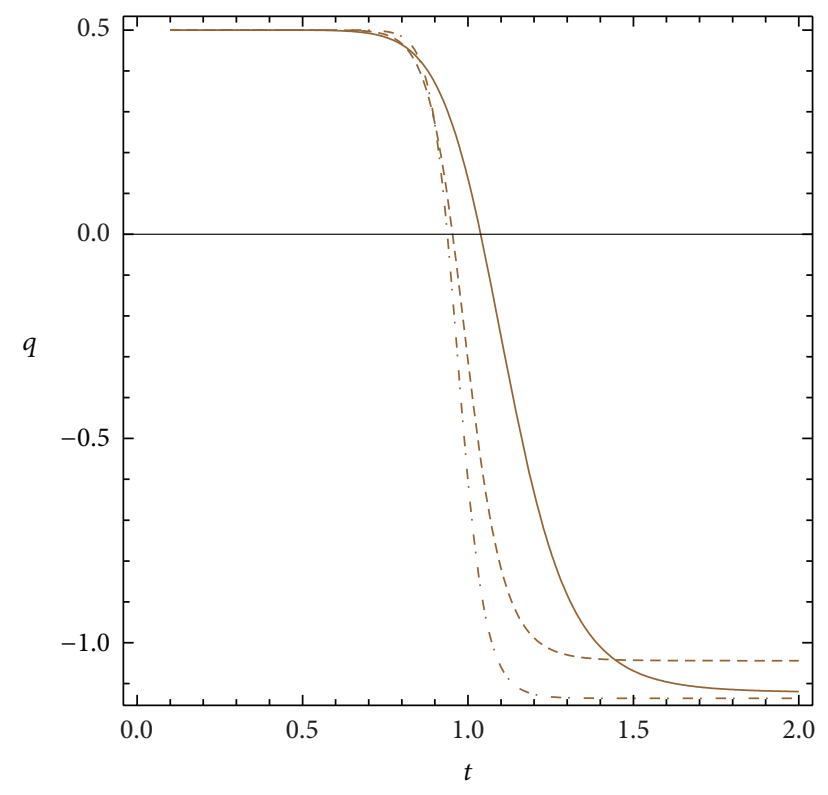

FIGURE 2: Evolution of deceleration parameter $q$ (21) against $t$. The solid, dot-dashed and dashed lines have the parameter combinations $\alpha=2.3,1.3,1.5, \beta=4.5,2.5,1.5, l_{1}=0.3,0.5,0.2$, and $l_{2}=4.5,6,7$.

3.2. Case II. In this section, we will only assume $a(t)=a_{0} t^{n}$ and $\phi(t)=\phi_{0} t^{m}$. We will use these ansatz to reconstruct the potential $V$ and the analytic function $f$ of the chameleon $\mathrm{BD}$ cosmology. On putting these ansatz in (8), we get an equation that involves $V, f$ and the remaining terms are functions of $t$

$$
\begin{aligned}
V= & -\frac{1}{a_{0}^{3}} \rho_{m 0} t^{-3 n} f \\
& +\frac{\phi_{0} t^{m-2}}{2}\left[-m^{2} \omega+6 n(m+n-(n-\beta) f)\right] .
\end{aligned}
$$

We again differentiate it with respect to $t$ and get a new equation involving $\dot{V}$ and $\dot{f}$. Using this in (9) along with the said ansatz, we get the following differential equation on $f$ with $t$ as the independent variable:

$$
\begin{aligned}
\frac{d f(t)}{d t}- & \frac{3(m+2 n)}{2 t} f(t) \\
= & 3 a_{0}^{3} \phi_{0} t^{m+3 n-1} \\
& \times(m n(t(3+2 \omega)-4-2 n) \\
& \left.\quad+m^{3}(1+\omega)-m^{2}(1+2 n)-4 n^{2}\right) \\
& \times\left(2\left(\rho_{m 0} t^{2}-a_{0}^{3}(m-2) \phi_{0}(n \alpha-\beta)\right)\right)^{-1} .
\end{aligned}
$$

Solving (23), we get reconstructed analytic function $f(\phi)$ as

$$
\begin{aligned}
f(\phi)= & f\left(\phi_{0} t^{m}\right) \\
= & t^{m+3 n} \\
& \times\left[C_{1} t^{m / 2}-\frac{2}{\rho_{m 0}\left(8+6 m+m^{2}\right) t^{2}}\right.
\end{aligned}
$$

$$
\begin{aligned}
& \times\{m n(4+m)(3+2 \omega) t \text { Hypergeometric } 2 F 1 \\
& \times\left[\frac{2+m}{4-2 m-6 n}, 1, \frac{6(n-1)+m}{2(3 n+m-2)}\right. \\
& \left.\quad \frac{a_{0}^{3}(m-2) \phi_{0}(n \alpha-\beta)}{\rho_{m 0}}\right] \\
& +(2+m)\left(-4 n^{2}-2 m n(2+n)\right. \\
& \left.-m^{2}(1+2 n)+m^{3}(1+\omega)\right)
\end{aligned}
$$

$\times$ Hypergeometric $2 F 1$

$$
\begin{gathered}
\times\left[\frac{4+m}{4-2 m-6 n}, 1, \frac{6 n-8+m}{2(3 n+m-2)},\right. \\
\left.\left.\left.\frac{a_{0}^{3}(m-2) \phi_{0}(n \alpha-\beta)}{\rho_{m 0}}\right]\right\}\right] .
\end{gathered}
$$

Using (24) in (22), we get the reconstructed potential function as

$$
\begin{aligned}
& V(\phi)=V\left(\phi_{0} t^{m}\right) \\
& =\frac{t^{-4+m}}{2 a_{0}^{3}(2+m)(4+m) \rho_{m 0}} \\
& \times\left(-(2+m)(4+m) \rho_{m 0} t^{2}\right. \\
& \times\left(2 C_{1} \rho_{m 0} t^{(4+m) / 2}+a_{0}^{3} \phi_{0}\right. \\
& \times(-6 m n+6 n \\
& \times\left(-n+C 1 t^{3 / 2(m+2 n)}(n \alpha-\beta)\right) \\
& \left.\left.\left.+m^{2} \omega\right)\right)\right) \\
& +4\left(\rho_{m 0} t^{2}+3 a_{0}^{3} n \phi_{0} t^{m+3 n}(n \alpha-\beta)\right) \\
& \times[m n(4+m)(3+2 \omega) t \text { Hypergeometric } 2 F 1 \\
& \times\left[1, \frac{2+m}{4-2 m-6 n}, 1+\frac{2+m}{4-2 m-6 n},\right. \\
& \left.\frac{a_{0}^{3}(m-2) \phi_{0}(n \alpha-\beta) t^{-2+m+3 n}}{\rho_{m 0}}\right] \\
& +(2+m)\left(-4 n^{2}-2 m n(2+n)\right. \\
& \left.-m^{2}(2 n+1)+m^{2}(1+\omega)\right)
\end{aligned}
$$

$\times$ Hypergeometric $2 F 1$

$$
\begin{gathered}
\times\left[1, \frac{4+m}{4-2 m-6 n}, 1+\frac{4+m}{4-2 m-6 n},\right. \\
\left.\left.\frac{a_{0}^{3}(m-2) \phi_{0}(n \alpha-\beta) t^{-2+m+3 n}}{\rho_{m 0}}\right]\right] .
\end{gathered}
$$




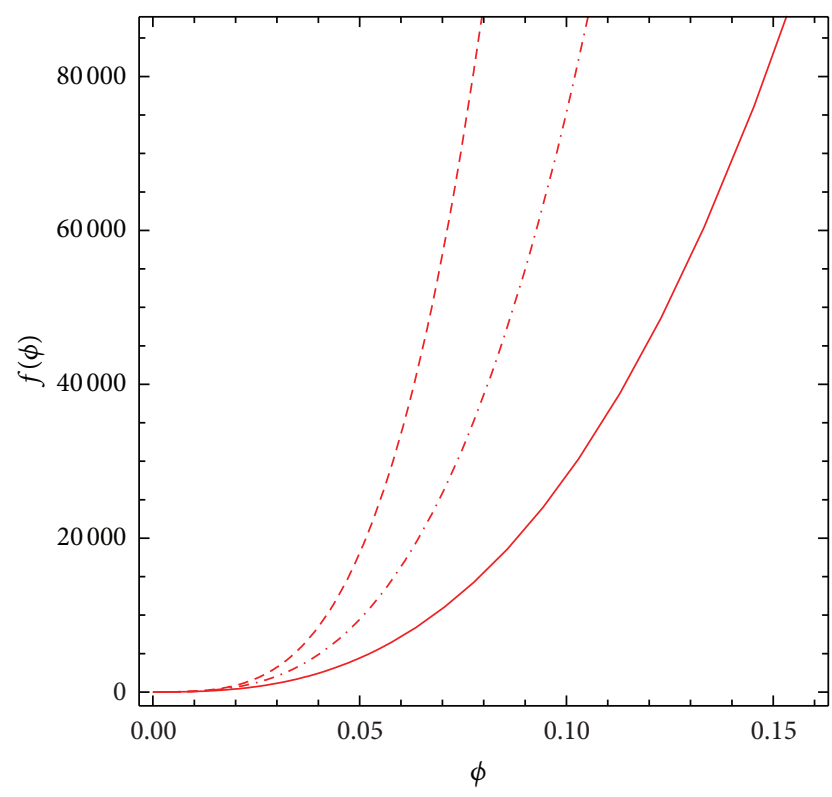

FIgURE 3: Reconstructed $f(\phi)$ against $\phi$ for various combinations of parameters.

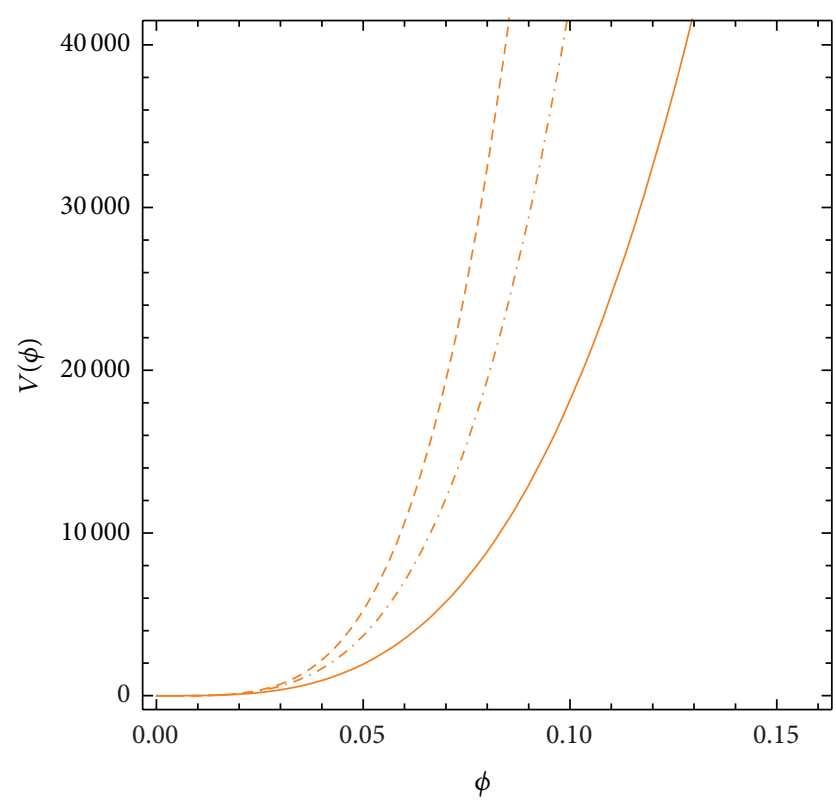

FIGURE 4: Reconstructed $V(\phi)$ against $\phi$ for various combinations of parameters.

In Figures 3, 4, and 5, we have plotted the reconstructed $f(\phi)$ against $\phi$, reconstructed $V(\phi)$ against $\phi$, and reconstructed $V(\phi)$ against $f(\phi)$, respectively. We observe that $f(\phi) \rightarrow 0$ as $\phi \rightarrow 0$ and $V(\phi) \rightarrow 0$ as $\phi \rightarrow 0$. These indicate the satisfaction of one of the sufficient conditions for realistic models. In Figure 5, we observe that the potential function $V(\phi)$ is increasing with the coupling function $f(\phi)$. Thus, we infer that as the matter-chameleon coupling gets stronger, the potential function also increases. In Figure 6, we have plotted $f(\phi)$ against $t$ for a range of positive values of $m$. This

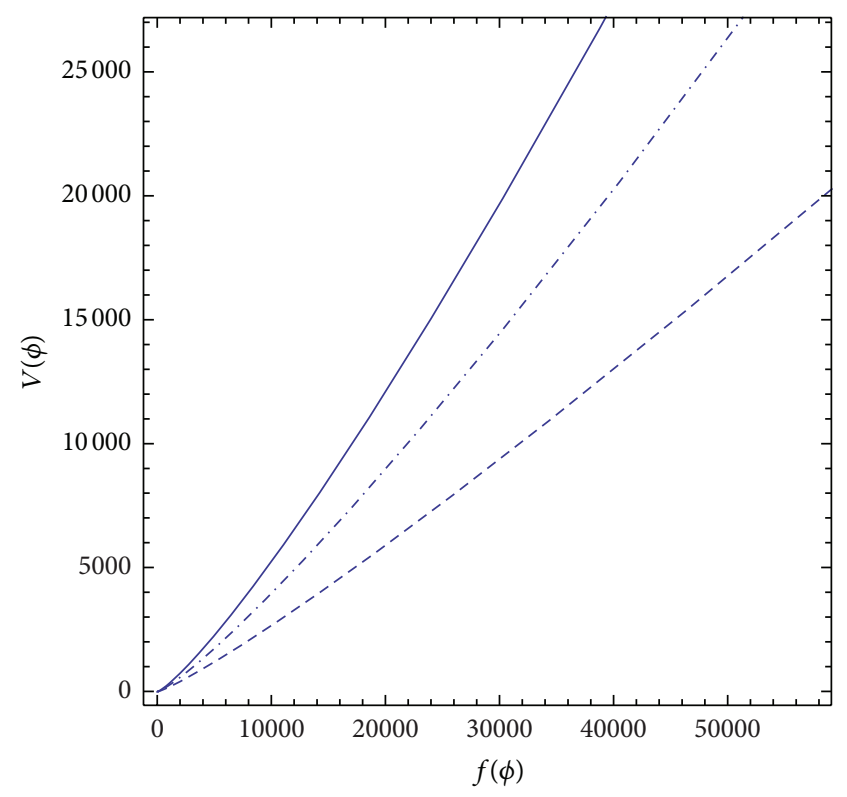

FIGURE 5: Reconstructed $V(\phi)$ against $f(\phi)$ for various combinations of parameters.

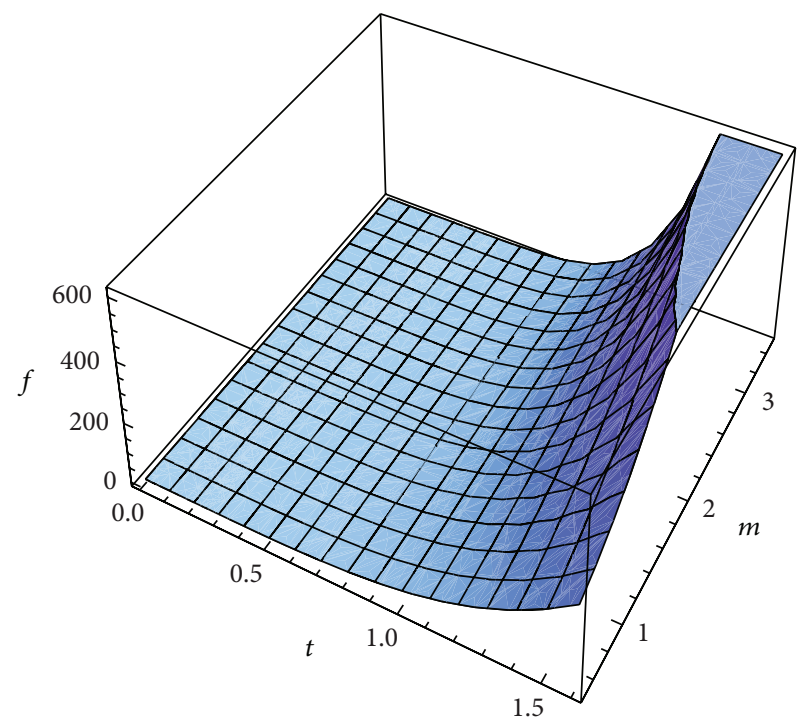

FiguRE 6: Reconstructed $f(\phi)$ against $t$ for a range of values of $m$.

figure shows that $f(\phi)$ in an increasing function of the cosmic time $t$. This indicates that with the expansion of the universe, the matter-chameleon coupling gets stronger, where the coupling function has been reconstructed for EHRDE.

\section{Conclusion}

In the present work, we have studied some features of the generalized $\mathrm{BD}$ model in which the scalar field is allowed to couple nonminimally with the matter sector. Extended holographic Ricci dark energy has been considered in the above framework of $\mathrm{BD}$ cosmology. The energy density has been considered in the form $\rho_{\Lambda}=3 \phi\left(\alpha H^{2}+\beta \dot{H}\right)$ to accommodate 
the BD scalar field in the dark energy density. The work has been carried out in two different perspectives. Firstly, we have assumed the ansatz $V(\phi)=V_{0} \phi^{l_{1}} ; f(\phi)=f_{0} \phi^{l_{2}} ; a(t)=a_{0} t^{n}$; and $\phi(t)=\phi_{0} t^{m}$ and derived some restrictions on the BD parameter $\omega$. Under this restriction, we have investigated the equation of state parameter for the dark energy and a quintom-like behavior has been observed. Furthermore, the deceleration parameter has shown a transition from decelerated to accelerated phase of the universe. In this scenario, it has been observed that the restrictions are leading to a stronger matter-chameleon coupling with the expansion of the universe. Secondly, we have considered only $a(t)=a_{0} t^{n}$ and $\phi(t)=\phi_{0} t^{m}$ and reconstructed the coupling function and potential for the extended holographic Ricci dark energy in $\mathrm{BD}$ model. We have observed that the potential is increasing with the increase in the matter-chameleon coupling. Furthermore, we have seen that for any positive value of $m, f(\phi)$ is an increasing function of $t$. This means that for this reconstruction, the coupling function is increasing; that is, the matterchameleon coupling is getting stronger with passage of cosmic time.

\section{Conflict of Interests}

The author declares that there is no conflict of interests regarding the publication of this paper.

\section{Acknowledgments}

The author wishes to acknowledge the financial support from the Department of Science and Technology, Government of India, under the Fast Track Scheme for Young Scientists. The Grant no. is SR/FTP/PS-167/2011. Also, the Visiting Associateship provided by the Inter-University Centre for Astronomy and Astrophysics (IUCAA), Pune, India, is duly acknowledged.

\section{References}

[1] A. G. Riess, V. Alexei Filippenko, P. M. Challis et al., "Observational evidence from supernovae for an accelerating universe and a cosmological constant," The Astronomical Journal, vol. 116, no. 3, p. 1009, 1998.

[2] S. Perlmutter, G. Aldering, G. Goldhaber et al., "Measurements of $\Omega$ and $\Lambda$ from 42 high-redshift supernovae," The Astrophysical Journal, vol. 517, no. 2, p. 565, 1999.

[3] P. J. E. Peebles and B. Ratra, "The cosmological constant and dark energy," Reviews of Modern Physics, vol. 75, no. 2, pp. 559606, 2003.

[4] E. J. Copeland, M. Sami, and S. Tsujikawa, "Dynamics of dark energy," International Journal of Modern Physics D, vol. 15, no. 11, pp. 1753-1935, 2006.

[5] T. Padmanabhan, "Dark energy: the cosmological challenge of the millennium," Current Science, vol. 88, p. 1057, 2005.

[6] T. Padmanabhan, "Dark energy and gravity," General Relativity and Gravitation, vol. 40, no. 2-3, pp. 529-564, 2008.

[7] L. Amendola and S. Tsujikawa, Dark Energy: Theory and Observations, Cambridge University Press, 2010.
[8] K. Bamba, S. Capozziello, S. I. Nojiri, and S. D. Odintsov, "Dark energy cosmology: the equivalent description via different theoretical models and cosmography tests," Astrophysics and Space Science, vol. 342, no. 1, pp. 155-228, 2012.

[9] J. Yoo and Y. Watanabe, "Theoretical models of dark energy," International Journal of Modern Physics D, vol. 21, no. 12, Article ID 1230002, 53 pages, 2012.

[10] S. Weinberg, "Search for dark matter," International Journal of Modern Physics A, vol. 23, no. 26, p. 4273, 2008.

[11] S. M. Carroll, "Quintessence and the rest of the world: suppressing long-range interactions," Physical Review Letters, vol. 81, no. 15, pp. 3067-3070, 1998.

[12] T. Chiba, T. Okabe, and M. Yamaguchi, "Kinetically driven quintessence," Physical Review D, vol. 62, no. 2, Article ID 023511, 8 pages, 2000.

[13] M. C. Bento, O. Bertolami, and A. A. Sen, "Generalized chaplygin gas, accelerated expansion, and dark-energy-matter unification," Physical Review D, vol. 66, no. 4, Article ID 043507, 5 pages, 2002.

[14] S. Nojiri and S. D. Odintsov, "Introduction to modified gravity and gravitational alternative for dark energy," International Journal of Geometric Methods in Modern Physics, vol. 4, no. 1, pp. 115-145, 2007.

[15] R. Myrzakulov, "Accelerating universe from $F(T)$ gravity," The European Physical Journal C, vol. 71, p. 1752, 2011.

[16] E. Elizalde, R. Myrzakulov, V. V. Obukhov, and D. Sáez-Gómez, " $\Lambda C D M$ epoch reconstruction from $F(R, G)$ and modified Gauss-Bonnet gravities," Classical and Quantum Gravity, vol. 27, no. 9, p. 095007, 2010.

[17] K. Bamba, R. Myrzakulov, S. Nojiri, and S. D. Odintsov, "Reconstruction of $\mathrm{f}(\mathrm{T})$ gravity: Rip cosmology, finite-time future singularities, and thermodynamics," Physical Review D, vol. 85, no. 10, p. 104036, 2012.

[18] R. Myrzakulov, "FRW cosmology in F(R,T) gravity”, The European Physical Journal C, vol. 72, p. 2203, 2012.

[19] S. Capozzielo, "Curvature quintessence," International Journal of Modern Physics D, vol. 11, no. 4, p. 483, 2002.

[20] L. Amendola, "Scaling solutions in general nonminimal coupling theories," Physical Review D, vol. 60, p. 043501, 1999.

[21] V. Sahni and Y. Shtanov, "Braneworld models of dark energy," Journal of Cosmology and Astroparticle Physics, no. 11, p. 014, 2003.

[22] S. Nojiri and S. D. Odintsov, "Unified cosmic history in modified gravity: from $\mathrm{F}(\mathrm{R})$ theory to Lorentz non-invariant models," Physics Reports, vol. 505, no. 2-4, pp. 59-144, 2011.

[23] C. Brans and R. H. Dicke, "Mach's principle and a relativistic theory of gravitation," Physical Review, vol. 124, no. 3, pp. 925935, 1961.

[24] G. S. Greenstein, "Brans-Dicke cosmology, I," The Astrophysical Journal Letters, vol. 1, p. 139, 1968.

[25] G. S. Greenstein, "Brans-Dicke cosmology, II," Astrophysics and Space Science, vol. 2, no. 2, pp. 155-165, 1968.

[26] M. K. Mak and T. Harko, "Brans-Dicke cosmology with a scalar field potential," Europhysics Letters, vol. 60, no. 1, pp. 155-161, 2002.

[27] A. E. Montenegro Jr. and S. Carneiro, "Exact solutions of BransDicke cosmology with decaying vacuum density," Classical and Quantum Gravity, vol. 24, no. 2, pp. 313-327, 2007.

[28] M. Arik and M. C. Calik, "Primordial and late-time inflation in Brans-Dicke cosmology," Journal of Cosmology and Astroparticle Physics, vol. 01, p. 013, 2005. 
[29] S. Sen and A. A. Sen, "Late time acceleration in Brans-Dicke cosmology," Physical Review D, vol. 63, no. 12, p. 124006, 2001.

[30] T. R. Seshadri, "Brans-Dicke class of cosmologies," Journal of Astrophysics and Astronomy, vol. 18, no. 4, pp. 339-342, 1997.

[31] S. Sen and T. R. Seshadri, "Self interacting Brans-Dicke cosmology and quintessence," International Journal of Modern Physics $D$, vol. 12 , no. 3 , p. 445,2003 .

[32] Y. Bisabr, "Cosmic acceleration in Brans-Dicke cosmology," General Relativity and Gravitation, vol. 44, no. 2, pp. 427-435, 2012.

[33] Y. Bisbar, "Chameleon Brans-Dicke cosmology," Physical Review D, vol. 86, no. 12, p. 127503, 2012.

[34] M. R. Setare, "The holographic dark energy in non-flat BransDicke cosmology," Physics Letters B, vol. 644, no. 2-3, pp. 99-103, 2007.

[35] J. Khoury and A. Weltman, "Chameleon cosmology," Physical Review D, vol. 69, no. 4, p. 044026, 2004.

[36] J. Khoury and A. Weltman, "Chameleon fields: awaiting surprises for tests of gravity in space," Physical Review Letters, vol. 93, no. 17, p. 171104, 2004.

[37] P. Brax, G. van de Bruck, A.-C. Davis, J. Khoury, and A. Weltman, "Detecting dark energy in orbit: the cosmological chameleon," Physical Review D, vol. 70, no. 12, p. 123518, 2004.

[38] H. Farajollahi and A. Salehi, "Dynamics of the self-interacting chameleon cosmology," Astrophysics and Space Science, vol. 338, no. 2, pp. 375-380, 2012.

[39] H. Farajollahi, A. Ravanpak, and G. F. Fadakar, "Holographic dark energy in chameleon tachyon cosmology," Astrophysics and Space Science, vol. 336, no. 2, pp. 461-467, 2011.

[40] F. Cannata and A. Y. Kamenshchikannata, "Chameleon cosmology model describing the phantom divide line crossing," International Journal of Modern Physics D, vol. 20, no. 2, p. 121, 2011.

[41] S. Das and N. Banerjee, "Brans-Dicke scalar field as a chameleon,” Physical Review D, vol. 78, no. 4, p. 043512, 2008.

[42] M. R. Setare and M. Jamil, "Holographic dark energy in BransDicke cosmology with chameleon scalar field," Physics Letters B, vol. 690, no. 1, pp. 1-4, 2010.

[43] A. Sheykhi and M. Jamil, "Interacting HDE and NADE in Brans-Dicke chameleon cosmology," Physics Letters B, vol. 694, no. 4-5, pp. 248-288, 2011.

[44] M. Jamil, I. Hussain, and D. Momeni, "Cosmic evolution in Brans-Dicke chameleon cosmology," The European Physical Journal Plus, vol. 126, p. 80, 2011.

[45] R. A. El-nabulsi, "Chamelionic generalized Brans-Dicke cosmology," The European Physical Journal Plus, vol. 127, p. 23, 2012.

[46] R. Bousso, "The holographic principle," Reviews of Modern Physics, vol. 74, no. 3, pp. 825-874, 2002.

[47] M. Li, "A model of holographic dark energy," Physics Letters B, vol. 603, no. 1-2, pp. 1-5, 2004.

[48] M. R. Setare and M. Jamil, "Holographic dark energy with varying gravitational constant in Hořava-Lifshitz cosmology," Journal of Cosmology and Astroparticle Physics, vol. 02, p. 010, 2010.

[49] M. Jamil, M. U. Farooq, and M. A. Rashid, "Generalized holographic dark energy model," The European Physical Journal C, vol. 61, no. 3, pp. 471-476, 2009.

[50] A. Sheykhi and M. Jamil, "Power-Law entropy corrected holographic dark energy model," General Relativity and Gravitation, vol. 43, no. 10, pp. 2661-2672, 2011.
[51] M. Jamil and M. U. Farooq, "Interacting holographic dark energy with logarithmic correction," Journal of Cosmology and Astroparticle Physics, vol. 03, p. 001, 2010.

[52] L. N. Granda and A. Oliveros, "Infrared cut-off proposal for the holographic density," Physics Letters B, vol. 669, no. 5, pp. 275$277,2008$.

[53] Y. U. Fei and Z. Jing-Fei, "Statefinder diagnosis for the extended holographic Ricci Dark energy model without and with interaction," Communications in Theoretical Physics, vol. 59, no. 2, p. 243,2013

[54] Wikipedia, 2013, http://en.wikipedia.org/wiki/Brans\%E2\%80\% 93Dicke_theory.

[55] V. Acquaviva, C. Baccigalupi, S. M. Leach, A. R. Liddle, and F. Perrotta, "Structure formation constraints on the Jordan-BransDicke theory," Physical Review D, vol. 71, no. 10, p. 104025, 2005. 

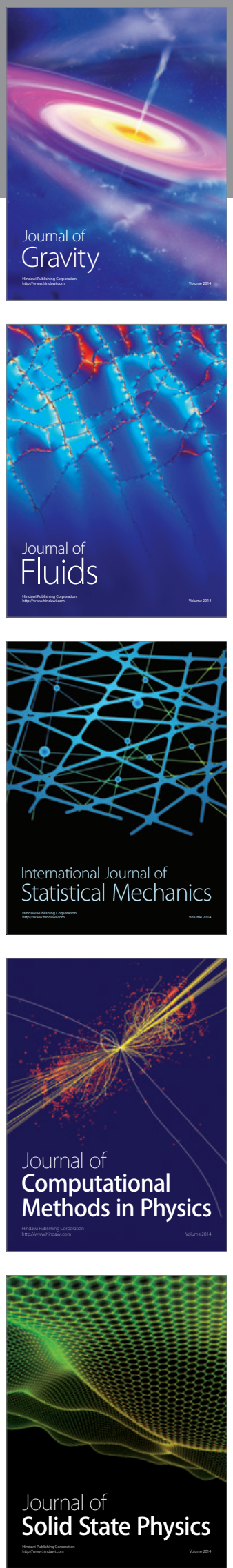

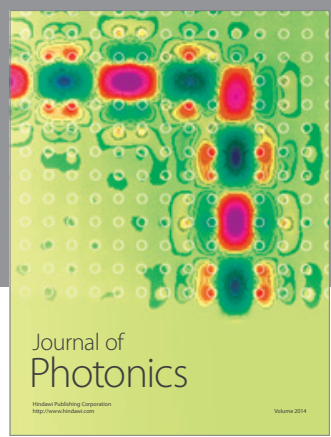

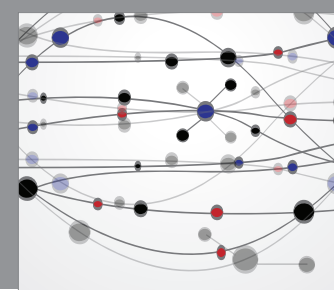

The Scientific World Journal

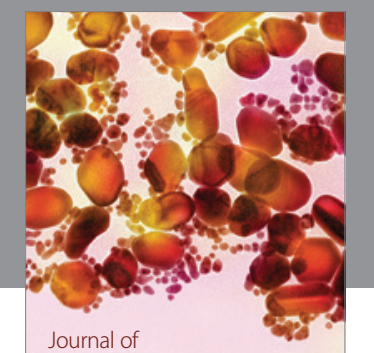

Soft Matter
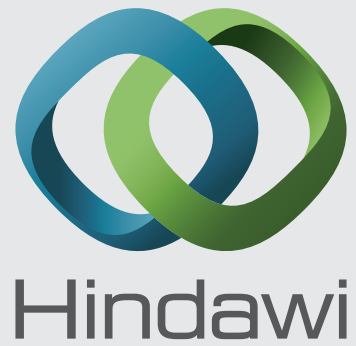

Submit your manuscripts at

http://www.hindawi.com
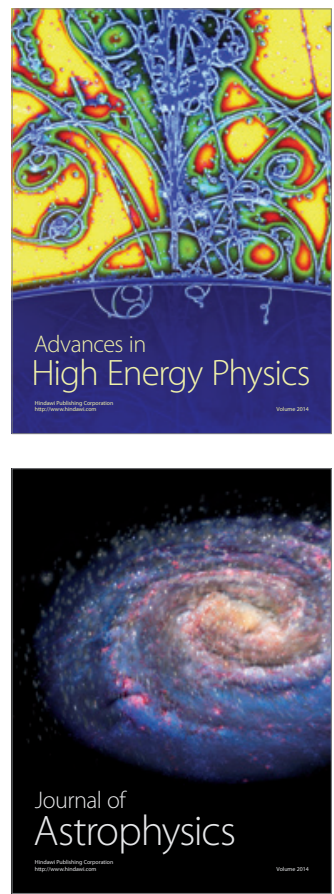
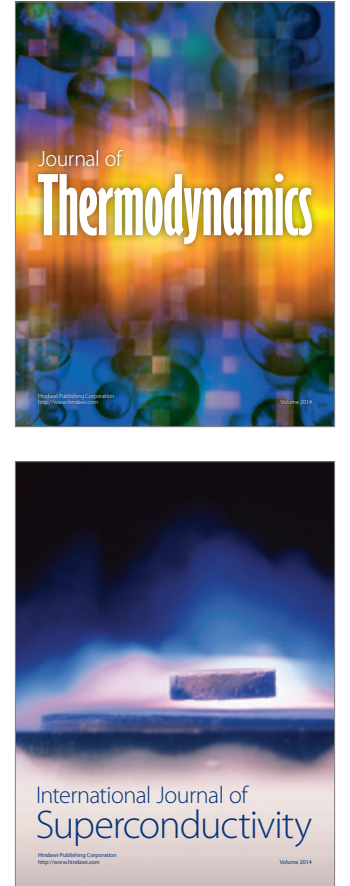
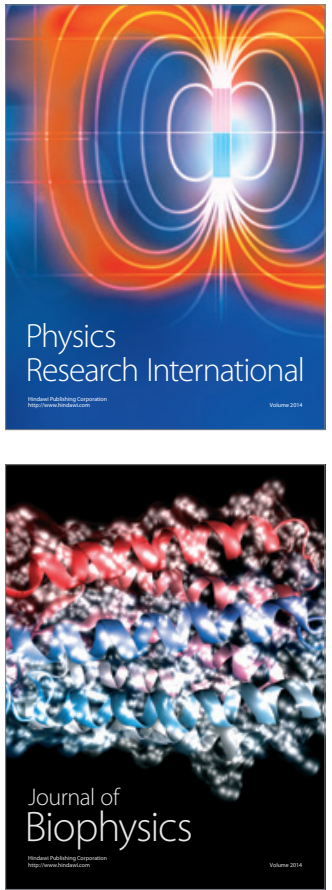
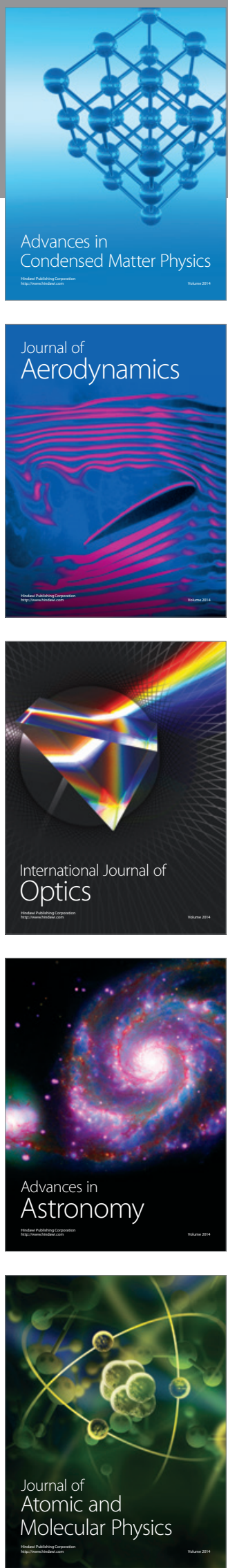